\title{
Plurality in multi-disciplinary research: Multiple institutional affiliations are associated with increased citations
}

\author{
Paul Sanfilippo ${ }^{\text {Corresp., } 1,2}$, Alex W Hewitt ${ }^{1,2,3}$, David A Mackey ${ }^{1,2,3}$ \\ 1 University of Melbourne, Royal Victorian Eye and Ear Hospital, Centre for Eye Research Australia, Melbourne, Australia \\ 2 University of Western Australia, Lions Eye Institute, Centre for Ophthalmology and Visual Science, Perth, Australia \\ 3 School of Medicine, University of Tasmania, Menzies Institute for Medical Research, Hobart, Tasmania, Australia \\ Corresponding Author: Paul Sanfilippo \\ Email address: prseye@gmail.com
}

Background. The institutional affiliations and associated collaborative networks that scientists foster during their research careers are salient in the production of high quality science. The phenomenon of multiple institutional affiliations and its relationship to research output remains relatively unexplored in the literature.

Methods. We examined 27,612 scientific articles, modelling the normalized citation counts received against the number of authors and affiliations held.

Results. In agreement with previous research, we found that teamwork is an important factor in high impact papers, with average citations received increasing concordant with the number of co-authors listed. For articles with more than five co-authors, we noted an increase in average citations received when authors with more than one institutional affiliation contributed to the research.

Discussion. Multiple author affiliations may play a positive role in the production of high-impact science. This 'roaming' behavior should be viewed by institutional boards as meritorious in the pursuit of scientific discovery. 
1

2 Plurality in multi-disciplinary research: Multiple institutional affiliations are

3 associated with increased citations

4

5 PG Sanfilippo PhD, ${ }^{1,2}$ AW Hewitt PhD FRANZCO, ${ }^{1,2,3}$ DA Mackey MD FRANZCO ${ }^{1,2,3}$

6 1. Centre for Ophthalmology and Visual Science, University of Western Australia, Lions Eye 7 Institute, Perth, Australia.

8 2. Centre for Eye Research Australia, University of Melbourne, Royal Victorian Eye and Ear 9 Hospital.

10 3. School of Medicine, Menzies Institute for Medical Research, University of Tasmania, 11 Hobart, Tasmania, Australia.

12

13 Word Count: 1690

14 Corresponding Author

15 Dr Paul Sanfilippo

16 Centre for Eye Research Australia

1732 Gisborne St, East Melbourne.

18 E-MAIL: prseye@gmail.com

19 


\section{Abstract}

21 Background. The institutional affiliations and associated collaborative networks that scientists 22 foster during their research careers are salient in the production of high quality science. The 23 phenomenon of multiple institutional affiliations and its relationship to research output remains 24 relatively unexplored in the literature.

25 Methods. We examined 27,612 scientific articles, modelling the normalized citation counts 26 received against the number of authors and affiliations held.

27 Results. In agreement with previous research, we found that teamwork is an important factor in 28 high impact papers, with average citations received increasing concordant with the number of co29 authors listed. For articles with more than five co-authors, we noted an increase in average citations 30 received when authors with more than one institutional affiliation contributed to the research.

31 Discussion. Multiple author affiliations may play a positive role in the production of high-impact 32 science. This increased researcher mobility should be viewed by institutional boards as meritorious 33 in the pursuit of scientific discovery. 


\section{Introduction}

With the Digital Revolution, the time-honoured model of scientific discovery being contingent on a singular intellect working independently of others, has expired. In the modern age of global travel and the interactive capabilities afforded by the internet, there is an expectation that good researchers are internationally mobile, both physically and virtually.(1) Researcher mobility is not a goal in itself, but rather a means of fostering collaborative networks at the many levels (e.g. institutional, interdisciplinary, international, etc.) that may drive successful scientific discovery. The increasing dominance of collaborative teams both within and between institutions has been documented to enhance efficiency and productivity as well as produce better science.(2) This is also reflected in the growth of international teams and their association with increased citation counts, a marker of research impact. $(2,3)$ Entangled within this collaborative research milieu, the institutional affiliations held by a researcher may also be viewed as a marker of capacity to facilitate knowledge exchange.(4) However, to date there has been little research from the burgeoning scientometric and bibliometric fields exploring the role of multiple institutional affiliations on scientific output.(5) To improve our understanding of this phenomenon, we conducted a large-scale analysis of scientific publications from four multi-disciplinary science journals (Science, Nature, Proceedings of the National Academy of Sciences [PNAS], PLOS Biology [PLOS]).

\section{Materials \& Methods}

We retrieved all 'articles' listed for the above journals from Web of Science (WoS) for the years 2010 - 2014, inclusive (search performed on 14/06/17). Articles were exported from WoS as BibTeX files, with complete metadata, then imported into the R statistical environment (6) for further processing. The bibliometrix package (7) was used to create a bibliographic data frame with cases (rows) corresponding to manuscripts and variables (columns) to Field Tags (metadata) in the original BibTex file. In this way the bibliographic attributes for each article (i.e. title, author's names, author's affiliations, citation count, document type, keywords, etc.) are formatted appropriately for subsequent analysis. The most important Field Tag for the purposes of this study is the Author Address (C1) tag which provides institutional address information for each author 
65 and where an author has multiple affiliations, lists these addresses separately. We split each 66 manuscript record by author name and affiliation address, with the sum of author name occurrences 67 indicating the number of distinct affiliations for that author. As comparisons of raw citation counts 68 are biased by virtue of time since publication (i.e. earlier publications have had longer to 69 accumulate citations), normalized citation counts were computed by dividing the raw value by the 70 number of days since June $30^{\text {th }}$ of the year of publication through to the search date $(14 / 06 / 17)$, 71 and then multiplying by 365.(8) This enables unbiased comparisons of citation counts irrespective 72 of the year of publication.

73

\section{Results and Discussion}

75 Of the 27,651 articles retrieved, 39 did not have affiliation data recorded and were excluded. The 76 total number of articles available for analysis was 27,612, with Science $(n=3,910)$, Nature $(n=$ $4,120)$, PNAS $(n=18,651)$, and PLOS $(n=931)$. The maximum number of citations for a single paper (published in 2012) was 4,143 (mean and median: 79.6 and 43.0, respectively). The maximum number of normalized citations was 828 , for the same paper (mean and median: 15.7 and 8.8, respectively). The maximum number of authors for a single paper was 2,908 (mean and median: 9.0 and 6.0, respectively), and the maximum number of author affiliations was 271 (mean and median: 4.7 and 4.0, respectively). Author affiliations were recorded as presented by WoS.

Table 1 shows the distribution of article and author appearances stratified by the number of author affiliations for the most- and least-cited articles split at the median normalized citation value (Highest Citations $=$ citations $>8.8[n=13,795]$, Lowest Citations $=$ citations $\leq 8.8[n=13,817])$. While the vast majority of author appearances were associated with only one institutional affiliation (74.1\%), 25.9\% of author appearances were linked with two (20.0\%) or more affiliation addresses. The maximum number of institutional affiliations held by an author was 12 . As these are non-independent observations, classical tests of contingency tables are not appropriate; however, one can easily appreciate the increased frequency of author appearances in the morecited publications. Indeed, the correlation between the normalized number of citations a paper received and the number of authors on that paper was statistically significant $(\rho=0.17, \mathrm{p}=<$ 
94 number of instiutional affiliations on that paper was $0.25, p=<0.001$. The correlation between

95 the number of authors and number of affiliations listed for each paper was greater, indicating closer 96 correspondence between the variables $(0.67, \mathrm{p}=<0.001)$.

97

98 To facilitate a simple yet fruitful investigation of the relationship between the number of 99 normalized citations a paper received and its association with authorship and affiliation frequency, we categorised the latter two variables. The number of authors attached to each paper was split into quartiles to create an 'Author Number' variable, with the following categories: $1=1-3$ authors/article, $2=4-5$ authors/article, $3=6-9$ authors/article, and $4=10-2,908$ authors/article.

103 Due to the low cell counts (Table 1) and to improve estimation in subsequent modelling, the maximum number of author affiliations held on a single paper was limited to six. This resulted in the exclusion of a further 47 papers, with 27,565 articles available for analysis. 'Maximum Affiliation' represents the maximum number of institutional affiliations held by a single author on an article. For example, if WoS listed an article with three authors each having two affiliations, and two authors each having three affiliations, in this case maximum affiliation would equal three. Table 2 shows the frequency distribution of articles by author number and maximum affiliation.

111 Figure 1 shows boxplots of citation counts for each category of author number and maximum 112 affiliation. There is a general trend of normalized citation count increasing across both factors. We 113 explored this relationship further in a linear regression model with normalized citation count as 114 the outcome, and author number and maximum affiliation as predictor variables (Supplementary 115 Table). Although these are technically count data, the mean citation value is high and the 116 distribution of the count model approximates the normal. Consequently, we have considered 117 citations a continous variable and utilised a linear model. We initially fit a model with an 118 interaction term (author number $\times$ maximum affiliation) and evaluated its signficance with a Wald 119 test. The resulting $p$-value was highly significant $(<0.001)$ suggesting the 15 coefficients for the 120 interaction terms are not simultaneously equal to zero, and an interaction effect exists between the 121 two variables (i.e. the relationship between maximum affiliation and citations received, varies 122 depending on the value of author number). The model was checked for multicollinearity using the 123 generalized variance inflation factor (GVIF). The raw output from the regression model are 124 supplied in the Supplementary Table. As interaction terms make coefficient interpretation difficult, 
125 results for the effect of each level of predictor are presented in a stratified manner, while holding 126 the other predictor constant (Table 3). In addition, we adjusted for year of publication and journal 127 in the analysis. It is of interest to note the effect of journal on normalized citation counts. Using 128 PNAS as the reference category journal (chosen as the most populous), both Science and Nature 129 receive on average higher normalized citation counts per paper $(\mathrm{p}<0.001)$ in comparison. 130 Citations received were not significantly different between PNAS and PLOS.

131

132 Table 3 shows the effect for each combination of maximum affiliation and author number on 133 normalized citation count. To further facilitate interpretation, we have limited maximum affiliation 134 data to four addresses. The effect size (Average Change in Normalised Citation Count) was 135 computed using a series of linear contrasts that enables the comparison of differences among 136 coefficients beyond the standard regression output. There are two main findings from these data:

137 first, the effect on citation count of an author holding more institutional affiliations increases as 138 the number of authors on a paper grows; and second, increasing the number of authors on a paper 139 tends to result in more citations received irrespective of the number of affiliations held.

140

141 When there are between 1 - 5 authors/article, increasing the number of affiliations an author holds 142 (relative to one) does not affect the average change in citation count. However, when there are 143 between 6 - 9 authors/article, authors with two institutional affiliations (relative to one) will, on 144 average, increase the citations a paper receives by $1.6(\mathrm{p}=0.006)$. This effect is even more 145 pronounced when there are more than 9 authors listed; here, citations increase on average by 2.3 $146(\mathrm{p}=0.002)$ for two affiliations, $5.8(\mathrm{p}<0.001)$ for three affiliations and $9.4(\mathrm{p}<0.001)$ for four 147 affiliations, relative to the reference group.

148

149 If we now interpret these effects while holding the number of affiliations constant, for researchers 150 with only one affiliation, increasing the number of authors on a paper results in a mean increase in 151 the citations received across all levels of author number (e.g. 6.5 for author number $=4$, relative 152 to $1, \mathrm{p}<0.001$ ). However, this effect remains significant for only greater author numbers (i.e. 4 vs 153 1) as the maximum number of affiliations held, increases. We would like to remind the reader that 
154 these data are cross-sectional in nature, and our discussion of 'effects' in the context of regression 155 analysis does not imply causation in the relationships explored.

156

\section{Conclusions}

158

159 These data align with previous observations in highlighting the increasing leverage of teamwork 160 in scientific research. $(2,3)$ They also serve to provide some insight into the relatively novel notion 161 that multiple author affiliations may play a positive role in the production of high-impact 162 science.(5) However, longitudinal analyses of citation count data would be necessary to explore 163 the basis for a causal relationship. To that end, further research is needed to address some of the 164 questions arising from the main finding of this study. What causes multi-institutional, larger 165 authored papers to have greater citation impact? Is increased institutional representation seminal 166 in the generation of high-quality science and therefore more highly cited works? Or are we 167 observing an artefact of highly-funded and highly-competitive research that by its nature will 168 generate more citations, irrespective of the number of authors or their affiliations. Clearly more 169 data is needed to comprehensively address these points. Until then, the holding of multiple 170 affiliations by authors should be viewed by institutional boards as a virtue and not a vice, as it 171 appears that greater researcher mobility may be advantageous to all.

172

173

174 


\section{References}

176

177 1. Schiermeier, Q. (2011). Career choices: The mobility imperative. Nature, 470 (7335), 563178564.

179 2. Wuchty S, Jones BF, \& Uzzi B (2007) The increasing dominance of teams in production of $180 \quad$ knowledge. in Science (American Association for the Advancement of Science), pp 1036$181 \quad 1039$.

182

183

184

185

186

187

188

189

190

191

192

193

194

195

196

3. Jones BF, Wuchty S, \& Uzzi B (2008) Multi-university research teams: shifting impact, geography, and stratification in science. in Science (American Association for the Advancement of Science), pp 1259-1262.

4. ESF (2013) New concepts of researcher mobility - a comprehensive approach including combined/part-time positions. Science Policy Briefing 49. Strasbourg: European Science Foundation.

5. Hottenrott H \& Lawson C (2017) A first look at multiple institutional affiliations: a study of authors in Germany, Japan and the UK. in Scientometrics (Springer Netherlands), pp 285-295.

6. Team RC (2017) R: A language and environment for statistical computing. $R$ Foundation for Statistical Computing, Vienna, Austria. URL http://www.r-project.org/.

7. Aria M \& Cuccurullo C (2016) bibliometrix: a R tool for comprehensive bibliometric

analysis of scientific literature. http://www.bibliometrix.org/).

8. Uddin, S., \& Khan, A. (2016). The impact of author-selected keywords on citation counts. Journal of Informetrics, 10(4), pp 1166-1177.

197

198 


\section{Figure 1 (on next page)}

Boxplots of citation counts stratified by author number and maximum affiliation.

The horizontal line and adjacent number indicate the median, the top and bottom of the boxes the interquartile range, and the number below each plot, the mean citation count. Citations are truncated at 500. 


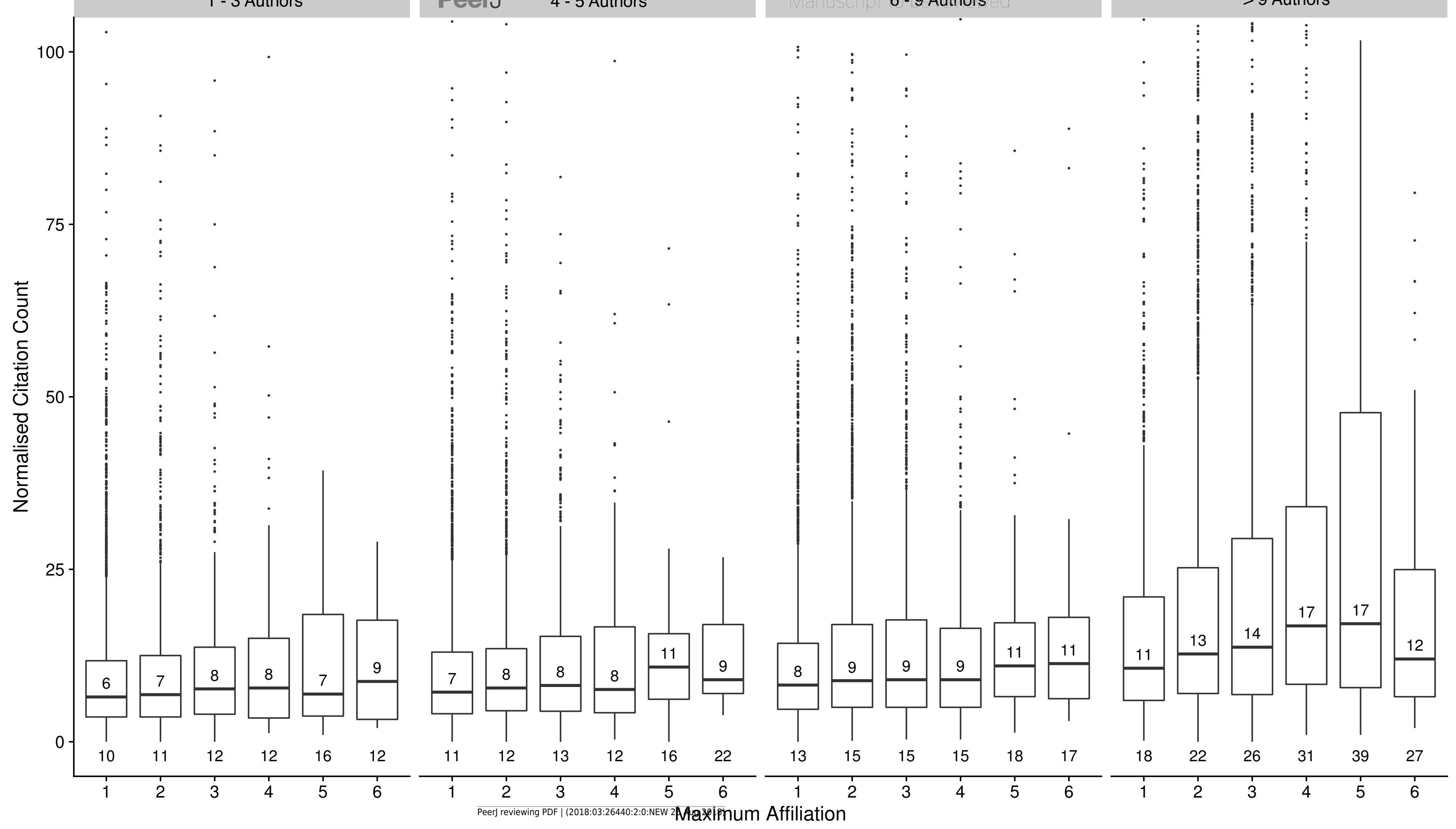




\section{Table $\mathbf{1}$ (on next page)}

Frequency distribution of articles and author appearances in most- and least-cited articles, stratified by the number of author affiliations attached to each article.

As individual articles may have contained multiple authors with different numbers of affiliations, they may appear more than once in the summary (i.e. an author may appear on multiple papers). 
Table 1: Frequency distribution of articles and author appearances in most- and least-cited articles, stratified by the number of author affiliations attached to each article. As individual articles may have contained multiple authors with different numbers of affiliations, they may appear more than once in the summary (i.e. an author may appear on multiple papers). Consequently, the values do not 4 represent unique numbers of articles or authors. Highest Citations $=$ normalized citations $>8.8$ [unique articles $=13,795]$, Lowest

5 Citations $=$ normalized citations $\leq 8.8$ [unique articles $=13,817])$.

\begin{tabular}{|c|c|c|c|c|c|}
\hline \multirow{2}{*}{$\begin{array}{l}\text { Number } \\
\text { of } \\
\text { Affiliation } \\
\text { s }\end{array}$} & \multicolumn{2}{|c|}{ Number of Article Appearances } & \multicolumn{3}{|c|}{ Number of Author Appearances } \\
\hline & $\begin{array}{c}\text { Lowest } \\
\text { Citations }\end{array}$ & Highest Citations & Lowest Citations (\%) & Highest Citations (\%) & Total (\%) \\
\hline 1 & 13102 & 13118 & $73430(29.4)$ & $111750(44.7)$ & $185180(74.1)$ \\
\hline 2 & 7327 & 8803 & $19174(7.7)$ & $30775(12.3)$ & $49949(20.0)$ \\
\hline 3 & 2451 & 3283 & $4381(1.7)$ & $6718(2.7)$ & $11099(4.4)$ \\
\hline 4 & 640 & 1027 & $1012(0.4)$ & $1622(0.7)$ & $2634(1.1)$ \\
\hline 5 & 185 & 319 & $304(0.1)$ & $457(0.2)$ & $761(0.3)$ \\
\hline 6 & 46 & 72 & $51(<0.1)$ & $109(<0.1)$ & $160(<0.1)$ \\
\hline 7 & 8 & 25 & $8(<0.1)$ & $29(<0.1)$ & $37(<0.1)$ \\
\hline 8 & 7 & 6 & $7(<0.1)$ & $7(<0.1)$ & $14(<0.1)$ \\
\hline 9 & 0 & 2 & 0 & $8(<0.1)$ & $8(<0.1)$ \\
\hline 10 & 0 & 1 & 0 & $1(<0.1)$ & $1(<0.1)$ \\
\hline 11 & 0 & 0 & 0 & 0 & 0 \\
\hline 12 & 0 & 1 & 0 & $2(<0.1)$ & $2(<0.1)$ \\
\hline
\end{tabular}


Total

98367 (39.4)

$151478(60.6)$

249845 (100) 


\section{Table 2 (on next page)}

Frequency distribution (\%) of articles in each category of author number and maximum affiliation.

Maximum Affiliation is the maximum number of affiliations held by a single author for each article, whilst the Author Number is the number of authors per article. 
1 Table 2: Frequency distribution (\%) of unique articles in each category of author number and 2 maximum affiliation. Maximum Affiliation is the maximum number of affiliations held by a single 3 author for each article, whilst the Author Number is the number of authors per article.

\begin{tabular}{|c|c|c|c|c|c|c|c|}
\hline \multicolumn{8}{|c|}{ Maximum Affiliation } \\
\hline Author Number & 1 & 2 & 3 & 4 & 5 & 6 & Total $(\%)$ \\
\hline \multirow{2}{*}{$1-3$} & 3142 & 1371 & 454 & 103 & 24 & 4 & 5098 \\
\hline & $(11.40)$ & $(4.97)$ & $(1.65)$ & $(0.37)$ & $(0.09)$ & $(0.01)$ & (18.49) \\
\hline \multirow{2}{*}{$4-5$} & 2715 & 2207 & 811 & 210 & 61 & 9 & 6013 \\
\hline & $(9.85)$ & $(8.01)$ & $(2.94)$ & $(0.76)$ & $(0.22)$ & $(0.03)$ & $(21.81)$ \\
\hline \multirow{2}{*}{$6-9$} & 2898 & 3845 & 1509 & 419 & 119 & 35 & 8825 \\
\hline & $(10.51)$ & $(13.95)$ & $(5.47)$ & $(1.52)$ & $(0.43)$ & $(0.13)$ & $(32.02)$ \\
\hline \multirow{2}{*}{$>9$} & 1387 & 3374 & 1859 & 695 & 250 & 64 & 7629 \\
\hline & $(5.03)$ & (12.24) & $(6.74)$ & $(2.52)$ & $(0.91)$ & $(0.23)$ & $(27.68)$ \\
\hline \multirow{2}{*}{ Total $(\%)$} & 10142 & 10797 & 4633 & 1427 & 454 & 112 & 27565 \\
\hline & $(36.79)$ & (39.17) & $(16.81)$ & $(5.18)$ & $(1.65)$ & $(0.41)$ & $(100.00)$ \\
\hline
\end{tabular}




\section{Table 3(on next page)}

Summary of regression model output for the effect of author number and maximum affiliation on average citation counts.

Within each stratum, the average change in citation count is relative to the first (reference) level. 


\begin{tabular}{|c|c|c|c|c|c|}
\hline \multirow[t]{3}{*}{ Covariate } & Effect & \multirow{3}{*}{$\begin{array}{c}\text { Average } \\
\text { Normalised } \\
\text { Citation Count }\end{array}$} & \multirow{3}{*}{$\begin{array}{l}\text { Average Change in } \\
\text { Normalised Citation } \\
\text { Count }\end{array}$} & \multirow{3}{*}{$\begin{array}{c}\text { 95\% C.I. for } \\
\text { Average } \\
\text { Change }\end{array}$} & \multirow[t]{3}{*}{$\mathrm{P}$} \\
\hline & & & & & \\
\hline & & & & & \\
\hline Author Number $=1$ & Max. Affiliation $=$ & 15.4 & 0 & & \\
\hline \multirow[t]{3}{*}{ (1-3 authors/article) } & 2 & 15.8 & 0.4 & $-1.1-1.9$ & 0.60 \\
\hline & 3 & 16.9 & 1.5 & $-0.8-3.8$ & 0.20 \\
\hline & 4 & 18.9 & 3.5 & $-1.1-8.0$ & 0.14 \\
\hline Author Number $=2$ & Max. Affiliation $=$ & 16.7 & 0 & & \\
\hline \multirow[t]{3}{*}{ (4-5 authors/article) } & 2 & 17.2 & 0.5 & $-0.8-1.8$ & 0.46 \\
\hline & 3 & 18.1 & 1.4 & $-0.4-3.2$ & 0.13 \\
\hline & 4 & 18.2 & 1.5 & $-1.8-4.8$ & 0.37 \\
\hline Author Number $=3$ & Max. Affiliation $=$ & 17.7 & 0 & & \\
\hline \multirow[t]{3}{*}{ (6-9 authors/article) } & 2 & 19.3 & 1.6 & $0.5-2.7$ & 0.006 \\
\hline & 3 & 19.7 & 2.0 & $0.5-3.4$ & 0.009 \\
\hline & 4 & 19.6 & 1.9 & $-0.5-4.3$ & 0.11 \\
\hline Author Number $=4$ & Max. Affiliation $=$ & 21.9 & 0 & & \\
\hline \multirow[t]{3}{*}{ (>9 authors/article) } & 2 & 24.2 & 2.3 & $0.8-3.7$ & 0.002 \\
\hline & 3 & 27.7 & 5.8 & $4.2-7.4$ & $<0.001$ \\
\hline & 4 & 31.3 & 9.4 & $7.2-11.5$ & $<0.001$ \\
\hline \multirow[t]{4}{*}{ Max. Affiliation $=1$} & Author Number $=1$ & 15.4 & 0 & & \\
\hline & 2 & 16.7 & 1.3 & $0.02-2.4$ & 0.05 \\
\hline & 3 & 17.7 & 2.3 & $1.1-3.5$ & $<0.001$ \\
\hline & 4 & 21.9 & 6.5 & $5.0-7.9$ & $<0.001$ \\
\hline \multirow[t]{4}{*}{ Max. Affiliation $=2$} & Author Number $=1$ & 15.8 & 0 & & \\
\hline & 2 & 17.2 & 1.4 & $-0.3-2.9$ & 0.10 \\
\hline & 3 & 19.3 & 3.5 & $2.1-4.9$ & $<0.001$ \\
\hline & 4 & 24.2 & 8.4 & $6.9-9.8$ & $<0.001$ \\
\hline \multirow[t]{4}{*}{ Max. Affiliation $=3$} & Author Number $=1$ & 17.0 & 0 & & \\
\hline & 2 & 18.1 & 1.1 & $-1.6-3.8$ & 0.42 \\
\hline & 3 & 19.7 & 2.7 & $0.3-5.2$ & 0.03 \\
\hline & 4 & 27.7 & 10.7 & $8.4-13.2$ & $<0.001$ \\
\hline Max. Affiliation $=4$ & Author Number $=1$ & 18.9 & 0 & & \\
\hline
\end{tabular}




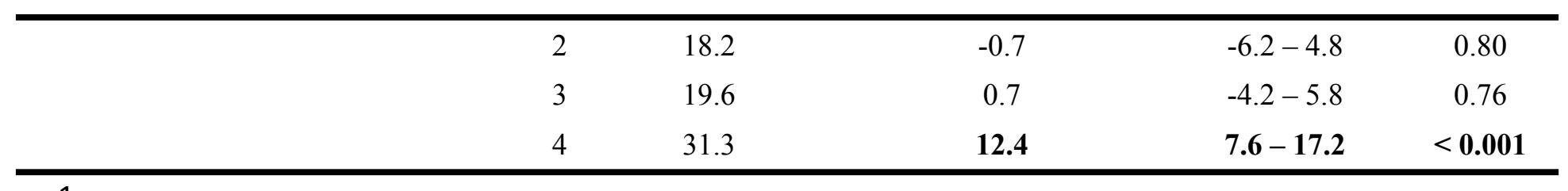

